\title{
Occurrence of Multinucleated Embryos After Intra- Cytoplasmic Sperm Injection Treatment May Be Prevented by the Administration of Prednisolone
}

\author{
Yuri Iwaki a, b, Miyako Funabiki ${ }^{\mathrm{a}, \mathrm{b}}$, Yoshihiro Tada ${ }^{\mathrm{a}}$, Masako Karita ${ }^{\mathrm{a}}$, Terumi Hayashi ${ }^{\mathrm{a}}$, \\ Takeharu Ota ${ }^{\text {a }}$,Kazunori Maeda ${ }^{\text {a }}$,Takashi Matsubara ${ }^{\text {a }}$, Yoshitaka Nakamura ${ }^{\text {a }}$, \\ Sagiri Taguchi ${ }^{a}, \mathrm{~b}, \mathrm{c}$
}

\begin{abstract}
The occurrence of multinucleated embryos after intra-cytoplasmic sperm injection (ICSI) treatment may be prevented by the administration of prednisolone.
\end{abstract}

Keywords: Infertility; Multinucleated embryos; Prednisolone; Pregnancy

\section{Introduction}

Multinucleated embryos after intra-cytoplasmic sperm injection (ICSI) treatment are often observed. Multinucleated embryos are associated with impaired cleavage and increased fragmentation and compromise the ongoing implantation rate [1]. However, a useful therapy for multinucleated embryos has not been confirmed. Prednisolone is a steroid that prevents the release of substances in the body that cause inflammation. Although prednisolone is used to treat many different inflammatory conditions, the efficacy of this steroid for multinucleated embryos is unclear.

\section{Case Report}

A 38-year-old woman with infertility visited our clinic in November 2013. She received seven ICSI treatments at our clinic, and prior to each treatment, she was administered clomifene (two tablets per day for 5 days), human menopausal gonadotropin and $0.25 \mathrm{mg}$ cetrotide (cetrorelix acetate for

\footnotetext{
Manuscript submitted December 11, 2017, accepted December 20, 2017
}

aIVF Center, Oak Clinic, Osaka, Japan

${ }^{\mathrm{b}}$ These authors contributed equally to this work.

${ }^{\mathrm{c} C}$ Corresponding Author: Sagiri Taguchi, IVF Center, Oak Clinic, 2-7-9 Tamade-Nishi, Nishinari-ku, Osaka 557-0045, Japan.

Email: taguchi_s@oakclinic-group.com injection). Although calcium ionophore treatment was administered after ICSI treatment, many of the embryos were multinucleated embryos (e.g., 3PN) that could not be transferred.

The patient was positive for anti-centromere antibody. Under the speculation that the existence of the antibody is related to the multinucleated embryos and it can be suppressed by prednisolone, prior to the eighth round of ICSI treatment, she was administered prednisolone (5 $\mathrm{mg}$ per day for 14 days) as well as clomifene (two tablets per day for 5 days), human menopausal gonadotropin, and $0.25 \mathrm{mg}$ cetrotide (cetrorelix acetate for injection).

As a result, all of the embryos had two pronuclei on the day after ICSI (Fig. 1a, b), and all of them were cryopreserved.
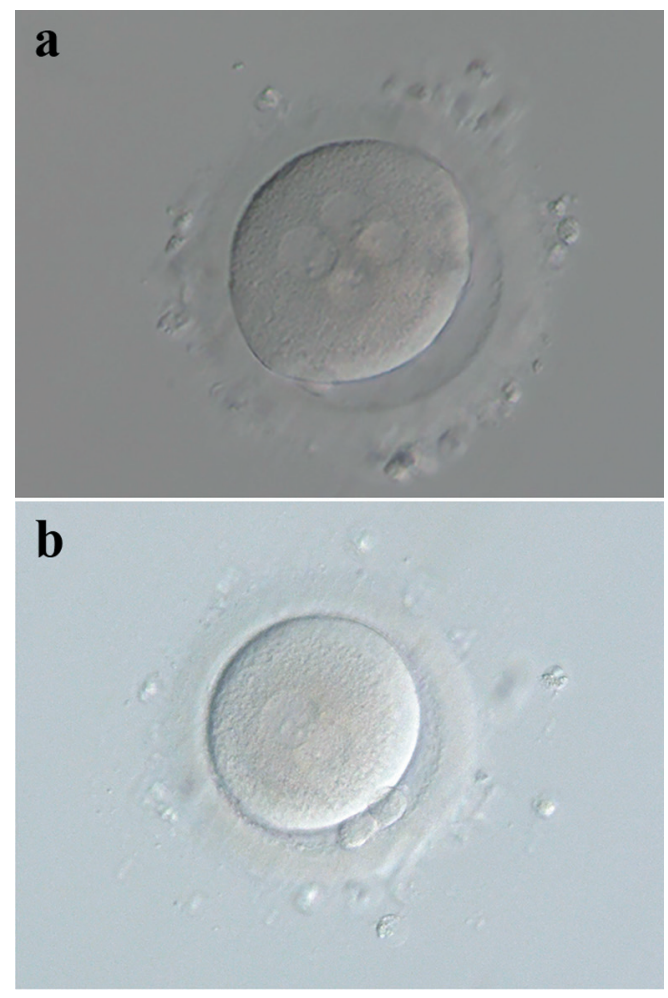

Figure 1. Efficacy of prednisolone for multinucleated embryos. (a) Before treatment of prednisolone. (b) After treatment of prednisolone. 
Furthermore, one of those four embryos (2PN) was later used for blastocyst transfer, and clinical pregnancy was confirmed by the observation of a gestational sac (GS) in July 2016. In addition, we confirm that the work in this study was conducted in accordance with the Code of Ethics of the World Medical Association (Declaration of Helsinki). Furthermore, all of the experiments were approved by the institutional review board of Oak Clinic in Japan.

\section{Discussion}

Multinucleation is a common phenomenon. It is seen in 74$79.4 \%$ of all cycles $[1,2]$. Furthermore, two reports showed that $31 \%$ of their embryos were multinucleated $[1,2]$. However, a useful therapeutic method for multinucleated embryos has not been confirmed. Therefore, under the speculation that the existence of antibody is related to the multinucleated embryos and that it can be suppressed by predonisolone, prior to the eighth round of ICSI treatment, we administered predonisolone ( $5 \mathrm{mg}$ per day for 14 days) to a patient. With the result of the case, the occurrence of multinucleated embryos after ICSI treatment was prevented by the administration of predonisolone. However, investigation of the detailed mechanism for the efficacy of predonisolone for multinucleated embryos is necessary in the near future.

\section{Acknowledgments}

We are grateful to all members at the Oak Clinic in Japan.

\section{Conflict of Interest}

We have no conflict of interest.

\section{Grant Support}

The present study is self-funded.

\section{References}

1. Van Royen E, Mangelschots K, Vercruyssen M, De Neubourg D, Valkenburg M, Ryckaert G, Gerris J. Multinucleation in cleavage stage embryos. Hum Reprod. 2003;18(5):1062-1069.

2. Jackson KV, Ginsburg ES, Hornstein MD, Rein MS, Clarke RN. Multinucleation in normally fertilized embryos is associated with an accelerated ovulation induction response and lower implantation and pregnancy rates in in vitro fertilization-embryo transfer cycles. Fertil Steril. 1998;70(1):60-66. 